\title{
Assessing markers of reproducibility and transparency in smoking cessation behaviour change intervention evaluations (2018-2019)
}

\author{
Emma Norris ${ }^{1,2 *}$, Yiwei He ${ }^{3}$, Rachel Loh ${ }^{3}$, Robert West ${ }^{4}$, Susan Michie ${ }^{1,3}$
}

*Corresponding author: Emma.Norris@brunel.ac.uk

${ }^{1}$ Centre for Behaviour Change; University College London, UK

${ }^{2}$ Department of Clinical Sciences; Brunel University, UK

${ }^{3}$ Psychology \& Language Sciences; University College London, UK

${ }^{4}$ Research Department of Epidemiology \& Public Health; University College London, UK

Keywords: reproducibility, transparency, meta-research, open science, smoking, behaviour change

\section{Abstract}

Introduction: Activities promoting research reproducibility and transparency are crucial. Evaluation of smoking cessation interventions is one area where vested interests may motivate reduced reproducibility and transparency.

Aims: Assess markers of transparency and reproducibility in smoking cessation behaviour change intervention evaluation reports.

Methods: One hundred evaluation reports of smoking cessation behaviour change intervention randomised controlled trials published in 2018-2019 were identified. Reproducibility markers of preregistration, protocol sharing, data-, materials- and analysis script-sharing, replication of a previous study and open access publication were coded in identified reports. Transparency markers of funding source and conflict of interest declarations were also coded. Coding was performed by two independent researchers, with inter-rater reliability calculated using Krippendorff's alpha.

Results: Seventy-one percent of reports were open access and 73\% pre-registered. However, only $13 \%$ provided accessible materials, $7 \%$ accessible data and $1 \%$ accessible analysis scripts. No reports 
were described as replication studies. Ninety-four percent of reports provided a funding source statement and eighty-eight percent of reports provided a conflict of interest statement.

Conclusions: Open data, materials, analysis and replications are rare in smoking behaviour change interventions, whereas funding source and conflict of interest declarations are common. Future smoking cessation research must be more reproducible to facilitate knowledge building. 


\section{Introduction}

Researchers are becoming increasingly aware of the importance of reproducibility and transparency in scientific research and reporting (Munafò et al., 2017; Nosek et al., 2015). A well-documented 'replication crisis' in psychology and other disciplines has shown that engrained academic incentives encouraging novel research has led to biased and irreproducible findings (loannidis, 2005; John, Loewenstein, \& Prelec, 2012; Nosek et al., 2012; Open Science Collaboration, 2015). Researchers, journals and funders across psychology and health sciences are now reforming scientific practice to improve the credibility and accessibility of research (Munafò et al., 2017; Norris \& O'Connor, 2019).

'Open Science', where some or all parts of the research process are made publicly and freely available, is essential for increasing research transparency, credibility, reproducibility and accessibility (Kathawalla, Silverstein \& Syed, 2020). Reproducibility-facilitating research behaviours are varied and occur throughout the research life-cycle. During study design; pre-registration and protocols specify the hypotheses, methods and analysis plan to be used in proposed subsequent research in repositories such as the Open Science Framework and AsPredicted. During data analysis, analysis scripts can be made more reproducible by marking their code with step-by-step comments to improve clarity and replication (van Vliet, 2020). During dissemination, the materials (such as intervention materials, questionnaires used), data and analysis scripts can be made available by uploading to repositories such as Open Science Framework or GitHub (Klein et al., 2018), facilitating the replication of effective research and interventions (Heirene, 2020). Allowing data and trial reports to be made available regardless of their findings enables a more accurate picture of the full state of research, minimising the 'file drawer' problem by which positive findings are more likely to be published than negative findings (Rotton, Foos, Van Meek \& Levitt, 1995). Transparencyfacilitating research behaviours include reporting sources of research funding and conflicts of interest (Fontanarosa, Flanagin, \& DeAngelis, 2005; Smith, 1998). These are important in that they 
help readers to make an informed judgement about potential risks of bias (Cristea \& loannidis, 2018).

Markers of reproducibility and transparency have been assessed in domains of psychology and life sciences. A recent study exploring 250 psychology studies published between 2014 and 2017 found transparency and reproducibility behaviours to be infrequent (Hardwicke et al., 2020). Although public availability of studies via open access was common (65\%), sharing of research resources was low for materials (14\%), raw data (2\%) and analysis scripts (1\%). Pre-registration (3\%) and study protocols (0\%) were also infrequent (Hardwicke et al., 2020). Transparency reporting was also inconsistent for funding statements (62\%) and conflict of interest disclosure statements (39\%) (Hardwicke et al., 2020). Meta-science studies have assessed reproducibility and transparency across other disciplines, such as social sciences (Hardwicke et al., 2019), biomedicine (Wallach et al., 2018) and biostatistics (Rowhani-Farid \& Barnett, 2018). Other research has focused on the prevalence of specific reproducibility behaviours, such as the prevalence of open access publications being around 45\% across scientific discipline assessed in 2015 (Piwowar et al., 2018).

However, the extent of reproducibility and transparency behaviours in public health research, including smoking cessation, is currently unclear. A recent investigation of randomised controlled trials addressing addiction found data sharing to be non-existent, with 0/394 trials making their data publicly available (Vassar, Jellison, Wendelbo, \& Wayant, 2020). Markers of wider reproducibility behaviours are yet to be assessed. Transparent reporting in terms of funding and conflicts of interest is especially crucial for smoking cessation, where tobacco and pharmaceutical companies fund some research directly or indirectly (Garne, Watson, Chapman, \& Byrne, 2005). Such vested interests may distort the reporting and interpreting of results and this may especially be the case in areas of controversy such e-cigarette research (Heirene, 2020; Munafò \& West, 2020; Smith, 1998; West, 
2020). The aim of the current study is to assess markers of i) reproducibility, and ii) transparency within smoking cessation intervention evaluation reports.

\section{Methods}

\section{Study design}

This was a retrospective observational study with a cross-sectional design. Sampling units were individual behaviour change intervention reports. This study applied a methodology used to assess reproducibility and transparency in the wider psychological sciences (Hardwicke et al., 2020) and social sciences (Harwicke et al., 2019). This study was pre-registered: https://osf.io/vqj5p.

\section{Sample of reports}

The Cochrane Tobacco Group Specialised Register of controlled trials was searched in November 2019, identifying 1630 reports from 2018 \& 2019. Inclusion criteria were randomised controlled trials published in 2018 and 2019, included in the Human Behaviour-Change Project (Michie et al., 2017; https://osf.io/efp4x/). Exclusion criteria were trial protocols, abstract-only entries and economic or process evaluations. Of the 157 reports remaining after applying these criteria, 100 reports were selected using a random number generator and PDFs obtained from journal websites.

\section{Measures}

Article characteristics recorded were: i) 2018 journal impact factor for each report using the Thomson Reuters Journal Citation Reports facility, and ii) country of the corresponding author (Table 1).

Markers of research reproducibility were assessed by recording presence of the following in included reports: i) Pre-registration: whether pre-registration was reported as carried out, where the pre- 
registration was hosted (e.g Open Science Framework, AsPredicted), whether it could be accessed and what aspects of the study were pre-registered; ii) Protocol sharing: whether a protocol was reported as carried out and what aspects of the study were included in the protocol; iii) Data sharing: whether data was available, where it was available (e.g online repository such as Open Science Framework, upon request from authors, as a journal supplementary file), whether the data was downloadable and accessible, whether data files were clearly documented and whether data files were sufficient to allow replication of reported findings; iv) Materials sharing: whether study materials were available, where they were available (e.g online repository such as Open Science Framework, upon request from authors, as a journal supplementary file) and whether the materials were downloadable and accessible; v) Analysis script-sharing: whether analysis scripts were available, where they were available (e.g online repository such as Open Science Framework, upon request from authors, as a journal supplementary file) and whether the analysis scripts were downloadable and accessible; vi) Replication of a previous study: whether the study claimed to be a replication attempt of a previous study; and vii) Open access publication: whether the study was published as open access.

Markers of research transparency was assessed by recording presence of the following in included reports: i) Funding sources: whether funding sources were declared and if research was funded by public organisations (such as research councils or charities), pharmaceutical, tobacco or other companies; ii) Conflicts of interest: whether conflicts of interest were declared and whether conflicts were with public organisations (such as research councils or charities), pharmaceutical, tobacco or other companies. All measured variables are shown in Table 1.

\section{Procedure}

Data collection took place between February and March 2020. Data for all measures were extracted onto a Google Form (https://osf.io/xvwjz/). All reports were independently coded by two 
researchers (YH \& RL). Any discrepancies were resolved through discussion, with input from a third researcher if required (EN).

\section{Analysis}

Research reproducibility was assessed using the markers of pre-registration, sharing of protocols, data, materials and analysis scripts, replication and open-access publishing (Table 1). Research transparency was assessed using the markers of funding source and conflicts of interest declarations. Inter-rater reliability of the independent coding of the two researchers was calculated using Krippendorff's alpha (Hayes \& Krippendorff, 2007) using Python 3.6 (https://github.com/HumanBehaviourChangeProject/Automation-InterRater-Reliability).

\section{Results}

Inter-rater reliability was assessed as excellent across all coding, $a=0.87$. Full data provided on OSF: https://osf.io/sw63b/.

\section{Sample characteristics}

Seventy-one out of 100 smoking cessation behaviour change intervention reports were published in 2018 and 29 published in 2019. Out of the 100 reports, four had no 2018 journal impact factor, with the remaining 96 reports having impact factors ranging from 0.888 to 70.67 (Mean $=4.95$ ). Fifty-four out of 100 reports took place in the United States of America (https://osf.io/i2zp3/). 


\section{Markers of reproducibility in smoking cessation behaviour change intervention evaluation} reports

Final reconciled coding of reproducibility and transparency for all smoking cessation behaviour change intervention reports can be found at: https://osf.io/jcgx6/.

\section{Article availability (open access)}

Seventy-one out of 100 smoking cessation behaviour change intervention reports were available via open access, with 29 were only accessible through a paywall (Figure 1A).

\section{Pre-registration}

Seventy-three out of 100 smoking cessation behaviour change intervention reports stated that they were pre-registered, with 72 of these being accessible. Fifty-five studies were pre-registered at ClinicalTrials.gov, eight at the International Standard Randomized Clinical Trial Number registry (ISRCTN), the Australian and New Zealand Clinical Trials Registry (ANZCTR), Chinese Clinical Trial

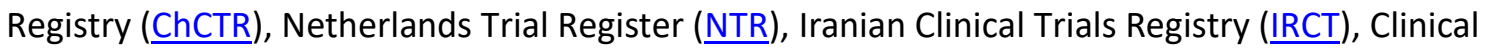
Research Information Service in Korea (RIS) or the UMIN Clinical Trials Registry in Japan (UMIN(TTR).

All of the 72 accessible pre-registrations reported methods, with 2 also reporting hypothesis. Only two accessible pre-registrations included hypothesis, methods and analysis plans. Twenty-six of the 100 reports did not include any statement of pre-registration. One report stated the study was not pre-registered (Figure 1B).

\section{Protocol availability}

Seventy-one out of 100 smoking cessation behaviour change intervention reports did not include a statement about protocol availability. For the 29 reports that included accessible protocols, 23 had a protocol that included hypothesis, methods and analysis plans. Three reports only had methods in 
their protocol, whereas two of them included both hypothesis and methods, and one of them included methods and analysis plans (Figure 1C).

\section{Material availability}

Twenty-two out of 100 reports included a statement saying the intervention materials used were available. Sixteen of these reports provided materials via journal supplementary files and six reports stated that their materials were only available upon request from the authors (Figure 1D).

\section{Data availability}

Sixteen out of 100 reports included a data availability statement. Nine reports stated data was available upon request from the authors and one stated the data was not available. The remaining six articles included their data in the supplementary files hosted by the journals, but one article's data file could not be opened. Four of the remaining articles had clearly documented data files, but only two of them contained all necessary raw data. As such in total, only seven reports provided links to data that was actually accessible (Figure 1E).

\section{Analysis script availability}

Three out of 100 reports included an analysis script availability statement. However only one provided accessible script as a supplementary file, with the remaining two stating analysis script was available upon request from authors (Figure 1F).

\section{Replication study}

None of the 100 smoking cessation behaviour change intervention reports were described as replication studies (Figure 1G). 


\section{Markers of transparency in smoking cessation behaviour change intervention evaluation reports}

Final reconciled coding of reproducibility and transparency markers for all smoking cessation behaviour change intervention reports can be found at: https://osf.io/icgx6/.

\section{Funding}

Ninety-four of the 100 smoking cessation behaviour change intervention reports included a statement about funding sources. Most of the reports disclosed public funding only such as via government-funded research grants, charities or universities $(n=80)$. Eight reports disclosed both public funding and funding from private companies. Five reports disclosed funding from private companies only, including pharmaceutical $(n=3)$, tobacco companies $(n=1)$ and other companies $(n=1)$. One report reported receiving no funding (Figure $1 \mathrm{H})$.

\section{Conflicts of interest}

Eighty-eight of the 100 articles provided a conflict of interest statement. Most of these reports reported that there were no conflicts of interests $(n=51)$. Thirty-seven reports reported that there was at least one conflict of interest, including from a pharmaceutical company ( $n=27)$, private company $(n=17)$, public organization $(n=13)$ and tobacco company $(n=3)$ (Figure 1l).

\section{Discussion}

This assessment of 100 smoking cessation behaviour change intervention evaluation reports identified varying levels of research reproducibility markers. Most reports were open access and preregistered; however, research materials, data and analysis scripts were not frequently provided and 
no replication studies were identified. Markers of transparency assessed here by funding source and conflicts of interest declarations were common.

\section{Assessment of reproducibility markers in smoking cessation behaviour change intervention evaluation reports}

Pre-registration, as a marker of research reproducibility, was found to be higher for smoking cessation interventions (73\%) than in wider psychological research (3\%) (Hardwicke et al., 2020). Open access reports were at similarly moderate levels (71\%) to psychology (65\%) (Hardwicke et al., 2020), but greater than the $45 \%$ observed in the social sciences (Hardwicke et al., 2019), $25 \%$ in biomedicine (Wallach et al., 2018) and 45\% across scientific literature published in 2015 (Piwowar et al., 2018). This high rate of open access publishing in smoking cessation interventions may reflect increasing requirements by health funding bodies for funded researchers to publish in open access outlets (Severin, Egger, Eve, \& Hürlimann, 2020; Tennant et al., 2016) and increasing usage of preprint publication outlets such as $\underline{\text { PsyArXiv }}$ for the psychological sciences and MedRxiv for medical sciences.

The rate of open materials was lower than in biomedicine (13\% vs 33\%) (Wallach et al., 2018) but similar to the social sciences rate of $11 \%$ (Hardwicke et al., 2019). Open analysis scripts were found to be as infrequently provided in smoking cessation interventions as in wider psychological research (both 1\%) (Hardwicke et al., 2020), social sciences (Hardwicke et al., 2019) and biostatistics (Rowhani-Farid \& Barnett, 2018).

Open data of smoking cessation interventions was found to be very low (7\%), but greater than the 0\% estimate in a larger sample of 394 smoking trials (Vassar et al., 2020) and to the $2 \%$ of wider psychological research (Hardwicke et al., 2020). Raw data are essential for meta-analyses to make sense of the diverse smoking cessation evidence. Common barriers for including studies in meta- 
analyses include a lack of available data, often after requests from authors (Greco, Zangrillo, BiondiZoccai, \& Landoni, 2013; loannidis, Patsopoulos, \& Rothstein, 2008). Provision of raw data as supplementary files to published intervention reports or via trusted third-party repositories such as the Open Science Framework (Klein et al., 2018) is important to facilitate evidence synthesis, especially in a field as important for global health as smoking cessation.

No replication attempts were identified in this sample of smoking cessation intervention reports, compared to $5 \%$ in wider psychology studies (Hardwicke et al., 2020) and $1 \%$ in the social sciences (Hardwicke et al., 2019). This lack of replication may be due to a lack of available resources of smoking interventions to facilitate replication, as identified in this study, or may reflect a lack of research prioritisation and funding for replication, with novel rather than confirmatory research prioritised at global, institutional levels (Munafò et al., 2017; Open Science Collaboration, 2015)

\section{Assessment of transparency markers in smoking cessation behaviour change intervention evaluation reports}

Declaration of funding sources and conflicts of interest, as markers of research transparency, were found here to be commonly provided in smoking cessation intervention evaluation reports. Funding sources were declared in more smoking cessation reports (95\%) than wider psychology (62\%) (Hardwicke et al., 2020), social sciences (31\%) (Hardwicke et al., 2019) and biomedical science reports (69\%) (Wallach et al., 2018). Similarly, a statement on conflicts of interest was provided more commonly in smoking cessation (88\%) than wider psychology (39\%) (Hardwicke et al., 2020), social sciences (39\%) (Hardwicke et al., 2019) and biomedical sciences reports (65\%) (Wallach et al., 2018). $17 \%$ of studies reported conflicts from private companies and $3 \%$ from tobacco companies. The comparatively high level of transparency markers observed here in smoking cessation interventions compared to other fields is likely to reflect improved reporting following previous 
controversies in the field (Bero, 2005; Garne et al., 2005; Malone \& Bero, 2003). Funding and disclosure statements are now commonly mandated by journals related to smoking cessation (Cristea \& Ioannidis, 2018; Munafò \& West, 2020; Nutu, Gentili, Naudet, \& Cristea, 2019).

\section{Strengths and limitations}

A strength of this study is its use of double-coding by two independent researchers of all reproducibility and transparency markers, enabling inter-rater reliability assessment. A limitation is that this study is based on a random sample of 100 evaluation reports of smoking cessation behaviour change interventions, whereby assessments of reproducibility and transparency may not be generalizable to broader smoking cessation interventions. Secondly, markers of reproducibility and transparency were dependent on what was described within evaluation reports. Direct requests to authors or additional wider searching of third-party registries such as Open Science Framework may have identified additional information indicating reproducibility.

\section{Future steps to increase reproducibility and transparency of smoking cessation interventions}

Urgent initiatives are needed to address the low levels of reproducibility markers observed here in smoking cessation research, especially in the areas of open materials, data, analysis scripts and replication attempts. As with any complex behaviour change, this transformation requires systems change across bodies involved in smoking cessation research: researchers, research institutions, funders, journals and beyond (Munafò et al., 2017; Norris \& O'Connor, 2019). Interventions are needed to increase the capability, opportunity and motivation of these bodies to facilitate behaviour change towards reproducible research in smoking cessation (Michie, van Stralen, \& West, 2011). For example, capability can be addressed by providing researcher training, equipping them with the skills needed to make their research open and reproducible, such as how to use the Open Science Framework, pre-print servers and how to make their analysis reproducible. Opportunity to engage in reproducible research in smoking cessation interventions can be facilitated within institutions, 
facilitating discussions around Open and reproducible working (Orben, 2019) and developing a culture around valuing progressive and open research behaviours (Norris \& O'Connor, 2019).

Motivation to research reproducibly can be addressed by providing researcher incentives (Norris \& O'Connor, 2019). Open Science badges recognising open data, materials and pre-registration have been adopted by journals as a simple, low-cost scheme to increase researcher motivation to engage in these reproducibility behaviours (Kidwell et al., 2016). Open Science badges have been identified as the only evidence-based incentive program associated with increased data sharing (RowhaniFarid, Allen, \& Barnett, 2017). However, although adoption of Open Science badges in smoking cessation journals is currently low, indicating this as one important initiative currently missing for journals in smoking cessation. Future research could compare this study's baseline assessment of reproducibility and transparency markers in smoking cessation intervention evaluation reports to assess changes in reporting and researcher behaviour.

\section{Conclusions}

Reproducibility markers of smoking cessation behaviour change intervention evaluation reports were varied. Pre-registration of research plans and open access publication were common, whereas the provision of open data, materials and analysis was rare and replication attempts were nonexistent. Transparency markers were common, with funding sources and conflicts of interest usually declared. Urgent initiatives are needed to improve reproducibility in open materials, data, analysis scripts and replication attempts. Future research can compare this baseline assessment of reproducibility and transparency in the field of smoking cessation to assess changes.

Acknowledgements. Thanks to Ailbhe N. Finnerty for calculating inter-rater reliability. 
Pre-registration: This study was pre-registered at: https://osf.io/yqj5p.

Data availability statement. All data are provided on OSF: https://osf.io/5rwsq/.

Financial support. Emma Norris was employed during this study on The Human Behaviour-Change Project, funded by a Wellcome Trust collaborative award (grant number 201,524/Z/16/Z).

Conflict of Interest. RW has undertaken research and consultancy for companies that develop and manufacture smoking cessation medications (Pfizer, J\&J and GSK). He is an unpaid advisor to The UK's National Centre for Smoking Cessation and Training and a Director of the not-for-profit Community Interest Company, Unlocking Behaviour Change Ltd. No other competing interests to disclose.

\section{References}

Bero, L. A. (2005). Tobacco industry manipulation of research. Public Health Reports, 120(2), 200.

Cristea, I.-A., \& loannidis, J. P. (2018). Improving disclosure of financial conflicts of interest for research on psychosocial interventions. JAMA Psychiatry, 75(6), 541-542.

Evangelou, E., Trikalinos, T. A., \& loannidis, J. P. A. (2005). Unavailability of online supplementary scientific information from articles published in major journals. The FASEB Journal, 19(14), 1943-1944. doi: 10.1096/fj.05-4784lsf

Fontanarosa, P. B., Flanagin, A., \& DeAngelis, C. D. (2005). Reporting conflicts of interest, financial aspects of research, and role of sponsors in funded studies. JAMA, 294(1), 110-111.

Garne, D., Watson, M., Chapman, S., \& Byrne, F. (2005). Environmental tobacco smoke research published in the journal Indoor and Built Environment and associations with the tobacco industry. The Lancet, 365(9461), 804-809.

Greco, T., Zangrillo, A., Biondi-Zoccai, G., \& Landoni, G. (2013). Meta-analysis: pitfalls and hints. Heart, Lung and Vessels, 5(4), 219-225.

Hardwicke, T. E., Thibault, R. T., Kosie, J., Wallach, J. D., Kidwell, M. C., \& loannidis, J. (2020). Estimating the prevalence of transparency and reproducibility-related research practices in psychology (2014-2017). https://osf.io/preprints/metaarxiv/9sz2y/ 
Hardwicke, T. E., Wallach, J. D., Kidwell, M. C., Bendixen, T., Crüwell, S., \& Ioannidis, J. P. (2019). An empirical assessment of transparency and reproducibility-related research practices in the social sciences (2014-2017). Royal Society Open Science, 7(2), 190806.

Hayes, A. F., \& Krippendorff, K. (2007). Answering the Call for a Standard Reliability Measure for Coding Data. Communication Methods and Measures, 1(1), 77-89.

Heirene, R. (2020). A call for replications of addiction research: Which studies should we replicate \& what constitutes a "successful" replication? Addiction Research \& Theory, DOI:

10.1080/16066359.2020.175113

loannidis, J. P. (2005). Why most published research findings are false. PLos Medicine, 2(8), e124.

loannidis, J. P., Patsopoulos, N. A., \& Rothstein, H. R. (2008). Reasons or excuses for avoiding metaanalysis in forest plots. BMJ, 336(7658), 1413-1415.

Iqbal, S. A., Wallach, J. D., Khoury, M. J., Schully, S. D., \& Ioannidis, J. P. (2016). Reproducible research practices and transparency across the biomedical literature. PLoS Biology, 14(1), e1002333.

John, L. K., Loewenstein, G., \& Prelec, D. (2012). Measuring the prevalence of questionable research practices with incentives for truth telling. Psychological Science, 23(5), 524-532.

Kathawalla, U. K., Silverstein, P., \& Syed, M. (2020). Easing Into Open Science: A Tutorial for Graduate Students. https://psyarxiv.com/vzjdp/

Kidwell, M. C., Lazarević, L. B., Baranski, E., Hardwicke, T. E., Piechowski, S., Falkenberg, L.-S., ... Hess-Holden, C. (2016). Badges to acknowledge open practices: A simple, low-cost, effective method for increasing transparency. PLoS Biology, 14(5).

Klein, O., Hardwicke, T. E., Aust, F., Breuer, J., Danielsson, H., Hofelich Mohr, A., . . Frank, M. C. (2018). A practical guide for transparency in psychological science. Collabra: Psychology, 4(1), 1-15.

Malone, R. E., \& Bero, L. (2003). Chasing the dollar: why scientists should decline tobacco industry funding: BMJ Publishing Group Ltd.

Michie, S., Thomas, J., Johnston, M., Mac Aonghusa, P., Shawe-Taylor, J., Kelly, M. P., . . Norris, E. (2017). The Human Behaviour-Change Project: harnessing the power of artificial intelligence and machine learning for evidence synthesis and interpretation. Implementation Science, 12(1), 121.

Michie, S., van Stralen, M. M., \& West, R. (2011). The behaviour change wheel: a new method for characterising and designing behaviour change interventions. Implement Science, 6, 42. doi: 10.1186/1748-5908-6-42

Munafò, M. R., Nosek, B. A., Bishop, D. V., Button, K. S., Chambers, C. D., Du Sert, N. P., . . loannidis, J. P. (2017). A manifesto for reproducible science. Nature Human Behaviour, 1(1), 1-9.

Munafò, M. R., \& West, R. (2020). E-cigarette research needs to adopt open science practices to improve quality. Addiction, 115(1), 3-4. 
Norris, E., \& O'Connor, D. B. (2019). Science as behaviour: Using a behaviour change approach to increase uptake of open science. Psychology \& Health, 34(12), 1397-1406.

Nosek, B.A., Alter, G., Banks, G.C., Borsboom, D., Bowman, S.D., Breckler, S.J., ... Yarkoni, T. (2015). Scientific standards. Promoting an open research culture. Science, 348 (6242), 1422-1425.

Nosek, B.A., Spies, J.R. \& Motyl, M. (2012). Scientific Utopia II: Restructuring incentives and practices to promote truth over publishability. Perspective on Psychological Science, 7(6), 615-631.

Nutu, D., Gentili, C., Naudet, F., \& Cristea, I. A. (2019). Open science practices in clinical psychology journals: An audit study. Journal of Abnormal Psychology, 128(6), 510.

Open Science Collaboration. (2015). Estimating the reproducibility of psychological science. Science, 349(6251), aac4716.

Orben, A. (2019). A journal club to fix science. Nature, 573(7775), 465-465.

Piwowar, H., Priem, J., Larivière, V., Alperin, J. P., Matthias, L., Norlander, B., . . Haustein, S. (2018). The state of OA: a large-scale analysis of the prevalence and impact of Open Access articles. PeerJ, 6, e4375.

Rotton, J., Foos, P.W., Van Meek, L., \& Levitt, M. (1995). Publication Practices and the File Drawer Problem: A Survey of Published Authors. Journal of Social Behavior and Personality, 10(1), 113.

Rowhani-Farid, A., Allen, M., \& Barnett, A. G. (2017). What incentives increase data sharing in health and medical research? A systematic review. Research Integrity and Peer Review, 2(1), 4. doi: 10.1186/s41073-017-0028-9

Rowhani-Farid, A., \& Barnett, A. G. (2018). Badges for sharing data and code at Biostatistics: an observational study. F1000Research, 7.

Severin, A., Egger, M., Eve, M. P., \& Hürlimann, D. (2020). Discipline-specific open access publishing practices and barriers to change: an evidence-based review. F1000Research, 7(1925), 1925.

Smith, R. (1998). Beyond conflict of interest: transparency is the key. BMJ, 317(7154), 291.

Tennant, J. P., Waldner, F., Jacques, D. C., Masuzzo, P., Collister, L. B., \& Hartgerink, C. H. (2016). The academic, economic and societal impacts of Open Access: an evidence-based review. F1000Research, 5.

van Vliet, M. (2020). Seven quick tips for analysis scripts in neuroimaging. PLOS Computational Biology, 16(3), e1007358.

Vassar, M., Jellison, S., Wendelbo, H., \& Wayant, C. (2020). Data sharing practices in randomized trials of addiction interventions. Addictive Behaviors, 102, 106193.

Wallach, J. D., Boyack, K. W., \& loannidis, J. P. (2018). Reproducible research practices, transparency, and open access data in the biomedical literature, 2015-2017. PLoS Biology, 16(11). 
REPRODUCIBILITY \& TRANSPARENCY OF SMOKING CESSATION INTERVENTIONS

West, R. (2020). Open science and pre-registration of studies and analysis plans. Addiction, 115(1), 55. 
Table 1. Measured variables and operationalization.

\begin{tabular}{|c|c|c|}
\hline Variables & Coder questions & Response options \\
\hline \multicolumn{3}{|c|}{$\begin{array}{l}\text { Coder instructions: To identify journal impact factors use the Thomson Reuters Journal Citation Reports } \\
\text { (https://library-guides.ucl.ac.uk/az.php?q=journal\%20citation\%20reports). } \\
\text { For country, check the institutional affiliation of the corresponding author. If there are multiple corresponding } \\
\text { authors, choose the first. If no corresponding author is identified, choose the first. If there are multiple } \\
\text { affiliations for the selected author, choose the first. }\end{array}$} \\
\hline Journal impact factor 2018 & $\begin{array}{l}\text { What is the } 2018 \text { journal } \\
\text { impact factor? }\end{array}$ & \\
\hline Country & $\begin{array}{l}\text { Which country is the } \\
\text { corresponding author based in } \\
\text { according to their affiliated? }\end{array}$ & [list countries]/ Unclear/ Other \\
\hline \multicolumn{3}{|c|}{ Reproducibility } \\
\hline \multicolumn{3}{|c|}{$\begin{array}{l}\text { Definitions: "Pre-registration" refers to the specification of important aspects of the study (typically } \\
\text { hypotheses, methods, and/or analysis plan) prior to commencement of the study. } \\
\text { Coder instructions: Check specific sections in the paper where these files might be located e.g., supplementary } \\
\text { materials, appendices, author notes, methods, and results sections. Search for "registration". }\end{array}$} \\
\hline Pre-registration statement & $\begin{array}{l}\text { Does the article state whether } \\
\text { or not the study (or some } \\
\text { aspect of the study) was pre- } \\
\text { registered? }\end{array}$ & $\begin{array}{l}\text { Yes - the statement says that } \\
\text { there was a pre-registration / } \\
\text { Yes - the statement says that } \\
\text { there was NO pre-registration / } \\
\text { No - there is no pre- } \\
\text { registration statement / Other* }\end{array}$ \\
\hline Pre-registration method & $\begin{array}{l}\text { Where does the article indicate } \\
\text { the pre-registration is located? }\end{array}$ & $\begin{array}{l}\text { Open Science Framework / } \\
\text { AsPredicted / } \\
\text { ClinicalTrials.gov / } \\
\text { AEA Trial Registry / } \\
\text { EGAP Registry / } \\
\text { Registered Report / Other* }\end{array}$ \\
\hline Pre-registration accessible & $\begin{array}{l}\text { Can you access and open the } \\
\text { pre-registration }\end{array}$ & Yes / No / Other* \\
\hline Pre-registration content & $\begin{array}{l}\text { What aspects of the study } \\
\text { appear to be pre-registered? } \\
\text { (select all that apply) }\end{array}$ & $\begin{array}{l}\text { Hypotheses } \\
\text { Methods } \\
\text { Analysis Plan } \\
\text { Other* }\end{array}$ \\
\hline \multicolumn{3}{|c|}{$\begin{array}{l}\text { Protocol sharing } \\
\begin{array}{l}\text { Definition: "protocol" refers to a document containing details about the study design, methods, and analysis } \\
\text { plan. It may or may not be pre-registered. }\end{array} \\
\text { Coder instructions: Search the article for the phrase 'protocol' and assess whether a link is provided to a } \\
\text { protocol document. }\end{array}$} \\
\hline Protocol availability & $\begin{array}{l}\text { Does the article link to an } \\
\text { accessible protocol? }\end{array}$ & Yes / No / Other* \\
\hline Protocol content & $\begin{array}{l}\text { What aspects of the study } \\
\text { appear to be included in the } \\
\text { protocol? (select all that apply) }\end{array}$ & $\begin{array}{l}\text { Hypotheses } \\
\text { Methods } \\
\text { Analysis Plan } \\
\text { Other* }\end{array}$ \\
\hline
\end{tabular}


availability statement" can be as simple as a url link to a data file, or as complex as a written explanation as to why data cannot be shared.

Coder instructions: Check the article for a data availability statement/link. They are often located in the "supplementary material", "acknowledgements", "author notes", "methods", or "results" sections. Search the article for the text "data availab" (to cover "data availability" and "data available").

\begin{tabular}{|c|c|c|}
\hline Data availability statement & $\begin{array}{l}\text { Does the article state whether } \\
\text { or not data are available? }\end{array}$ & $\begin{array}{l}\text { Yes - the statement says that } \\
\text { the data (or some of the data) } \\
\text { are available / } \\
\text { Yes - the statement says that } \\
\text { the data are NOT available / } \\
\text { No - there is no data availability } \\
\text { statement / Other* }\end{array}$ \\
\hline Data sharing method & $\begin{array}{l}\text { How does the statement } \\
\text { indicate the data are available? }\end{array}$ & $\begin{array}{l}\text { Upon request from the authors } \\
\text { / Personal or institution } \\
\text { website / } \\
\text { An online, third-party } \\
\text { repository (e.g., OSF, FigShare } \\
\text { etc.) / } \\
\text { supplementary materials } \\
\text { hosted by the journal / } \\
\text { Other* }\end{array}$ \\
\hline Data accessibility & $\begin{array}{l}\text { Can you access, download, and } \\
\text { open the data files? }\end{array}$ & Yes / No / Other* \\
\hline Data documentation & $\begin{array}{l}\text { Are the data files clearly } \\
\text { documented? }\end{array}$ & Yes / No / Other* \\
\hline Data content & $\begin{array}{l}\text { Do the data files appear to } \\
\text { contain all of the raw data } \\
\text { necessary to reproduce the } \\
\text { reported findings? }\end{array}$ & Yes / No / Unclear / Other* \\
\hline
\end{tabular}

\section{Materials sharing}

Definitions: "materials" refers to any study items that would be needed to repeat the study, such as stimuli, survey instruments, and computer code/software used for data collection, presentation stimuli or running experiments

\begin{tabular}{|l|l|l|}
\hline Materials availability statement & $\begin{array}{l}\text { Does the article state whether } \\
\text { or not materials are available? }\end{array}$ & $\begin{array}{l}\text { Yes - the statement says that } \\
\text { the materials (or some of the } \\
\text { materials) are available / } \\
\text { Yes - the statement says that } \\
\text { the materials are NOT available } \\
/\end{array}$ \\
Materials sharing method & $\begin{array}{l}\text { No - there is no materials } \\
\text { according to the statement, } \\
\text { accessible? } \\
\text { Ore the materials }\end{array}$ & $\begin{array}{l}\text { Upon request from the authors } \\
\text { / Personal or institution } \\
\text { website / } \\
\text { An online, third-party } \\
\text { repository (e.g., OSF, FigShare } \\
\text { etc.) / } \\
\text { supplementary materials } \\
\text { hosted by the journal / } \\
\text { Other* }\end{array}$ \\
\hline
\end{tabular}




\begin{tabular}{|c|c|c|}
\hline Materials accessibility & $\begin{array}{l}\text { Can you access, download, and } \\
\text { open the materials files? }\end{array}$ & Yes / No / Other* \\
\hline \multicolumn{3}{|c|}{$\begin{array}{l}\text { Definition: "Analysis scripts" refers to specification of data preparation and analysis steps in the form of highly } \\
\text { detail step-by-step instructions for using point-and-click software, analysis code (e.g., R), or syntax (e.g., from } \\
\text { SPSS). } \\
\text { Coder instructions: Check the article for an analysis script availability statement/link. They are often located in } \\
\text { the "supplementary material", "acknowledgements", "author notes", "methods", or "results" sections. Search } \\
\text { for the text "analysis script" and "analysis code". }\end{array}$} \\
\hline $\begin{array}{l}\text { Analysis script availability } \\
\text { statement }\end{array}$ & $\begin{array}{l}\text { Does the article state whether } \\
\text { or not analysis scripts are } \\
\text { available? }\end{array}$ & $\begin{array}{l}\text { Yes - the statement says that } \\
\text { the analysis scripts (or some of } \\
\text { the analysis scripts) are } \\
\text { available / } \\
\text { Yes - the statement says that } \\
\text { the analysis scripts are NOT } \\
\text { available / } \\
\text { No - there is no analysis script } \\
\text { availability statement }\end{array}$ \\
\hline Analysis script sharing method & $\begin{array}{l}\text { According to the statement, } \\
\text { how are the analysis scripts } \\
\text { accessible? }\end{array}$ & $\begin{array}{l}\text { Upon request from the authors } \\
\text { / Personal or institution } \\
\text { website / } \\
\text { An online, third-party } \\
\text { repository (e.g., OSF, FigShare } \\
\text { etc.) / } \\
\text { supplementary materials } \\
\text { hosted by the journal / } \\
\text { Other* }\end{array}$ \\
\hline Analysis script accessibility & $\begin{array}{l}\text { Can you access, download, and } \\
\text { open the analysis script files? }\end{array}$ & Yes / No / Other* \\
\hline \multicolumn{3}{|c|}{$\begin{array}{l}\text { Replication } \\
\text { Definitions: "replication" refers to repetition of a previous study's methods in order to ascertain whether } \\
\text { similar findings can be obtained. } \\
\text { Coder instructions: Search the abstract and introduction for the phrase "replicat" (to cover 'replication', } \\
\text { 'replicates' etc). Confirm the authors are using the phrase with the definition provided above. }\end{array}$} \\
\hline Replication statement & $\begin{array}{l}\text { Does the article claim to report } \\
\text { a replication study? }\end{array}$ & $\begin{array}{l}\text { The article claims to report a } \\
\text { replication study (or studies) / } \\
\text { There is no clear statement that } \\
\text { the article reports a replication } \\
\text { study (or studies) } \\
\text { / Other* }\end{array}$ \\
\hline \multicolumn{3}{|c|}{$\begin{array}{l}\text { Open access } \\
\text { Coder instructions: To establish the open access status of the article: Go to https://openaccessbutton.org/ } \\
\text { and enter the article's doi (e.g., "10.1371/journal.pcbi.1004574") if available (if not, enter the article title). If a } \\
\text { link is provided, check that you can access the article at the link. If the article is accessible answer "Yes". If the } \\
\text { article is not accessible at the provided link, or no link is provided, answer "No". }\end{array}$} \\
\hline Open access status & Is the article open access? & $\begin{array}{l}\text { Yes - found via open access } \\
\text { button / Yes - found via other } \\
\text { means / No - could not access } \\
\text { article other than through } \\
\text { paywall / Other* }\end{array}$ \\
\hline
\end{tabular}




\section{Transparency \\ Funding}

Coder instructions: Funding is usually reported in a specific section e.g., "Author information", or "Funding statement". Search the article for the phrase "funding". If you are unsure whether an organisation is a tobacco company, pharmaceutical company, other private company or public organisation, Google the organisation name and code accordingly. If it is unclear to you whether the funding is private or public, choose the 'other' option and enter 'unclear'.

\begin{tabular}{|l|l|l}
\hline Funding statement & $\begin{array}{l}\text { Does the article include a } \\
\text { statement indicating whether } \\
\text { there were funding sources? }\end{array}$ & $\begin{array}{l}\text { Yes - the statement says that } \\
\text { there was funding from a } \\
\text { tobacco company (e.g Phillip } \\
\text { Morris, British American } \\
\text { Tobacco, China Tobacco, } \\
\text { Imperial Brands) / } \\
\text { Yes - funding from a } \\
\text { pharmaceutical company (e.g } \\
\text { Pfizer, GSK)/ } \\
\text { Yes - funding from another } \\
\text { private company / } \\
\text { Yes - funding from a public } \\
\text { organisation (e.g National } \\
\text { Institute of Health Research)/ } \\
\text { Yes - the statement says that } \\
\text { there was no funding was } \\
\text { provided / } \\
\text { No - there is no funding } \\
\text { statement / } \\
\text { Unclear/Other* }\end{array}$ \\
\end{tabular}

\section{Conflict of interest}

Coder instructions: Conflicts of interest are usually reported in a specific section e.g. "Author information" or "Conflict of interest statement". Search the article for the phrases "conflict of interest" and/or "competing interest". If you are unsure whether an organisation is a tobacco company, pharmaceutical company, other private company or public organisation, Google the organisation name and code accordingly. If it is unclear to you whether the funding is private or public, choose the 'other' option and enter 'unclear'.

\begin{tabular}{|l|l|l} 
Conflict of Interest statement & $\begin{array}{l}\text { Does the article include a } \\
\text { statement indicating whether } \\
\text { there were any conflicts of } \\
\text { interest? }\end{array}$ & $\begin{array}{l}\text { Yes - the statement says that } \\
\text { there was a conflict of interest } \\
\text { from a tobacco company / } \\
\text { Yes - conflict of interest from a } \\
\text { pharmaceutical company / } \\
\text { Yes - conflict of interest from } \\
\text { another private company / } \\
\text { Yes - conflict of interest from a } \\
\text { public organisation (e.g } \\
\text { National Institute of Health } \\
\text { Research)/ } \\
\text { Yes - the statement says that } \\
\text { there is no conflict of interest / } \\
\text { No - there is no conflict of } \\
\text { interest statement / } \\
\text { Other* }\end{array}$
\end{tabular}

*If a response marked with an asterisk is selected, the coder is asked to provide more detail in a free-text response box. 
Figure 1. Overview of reproducibility and transparency markers in smoking cessation interventions.
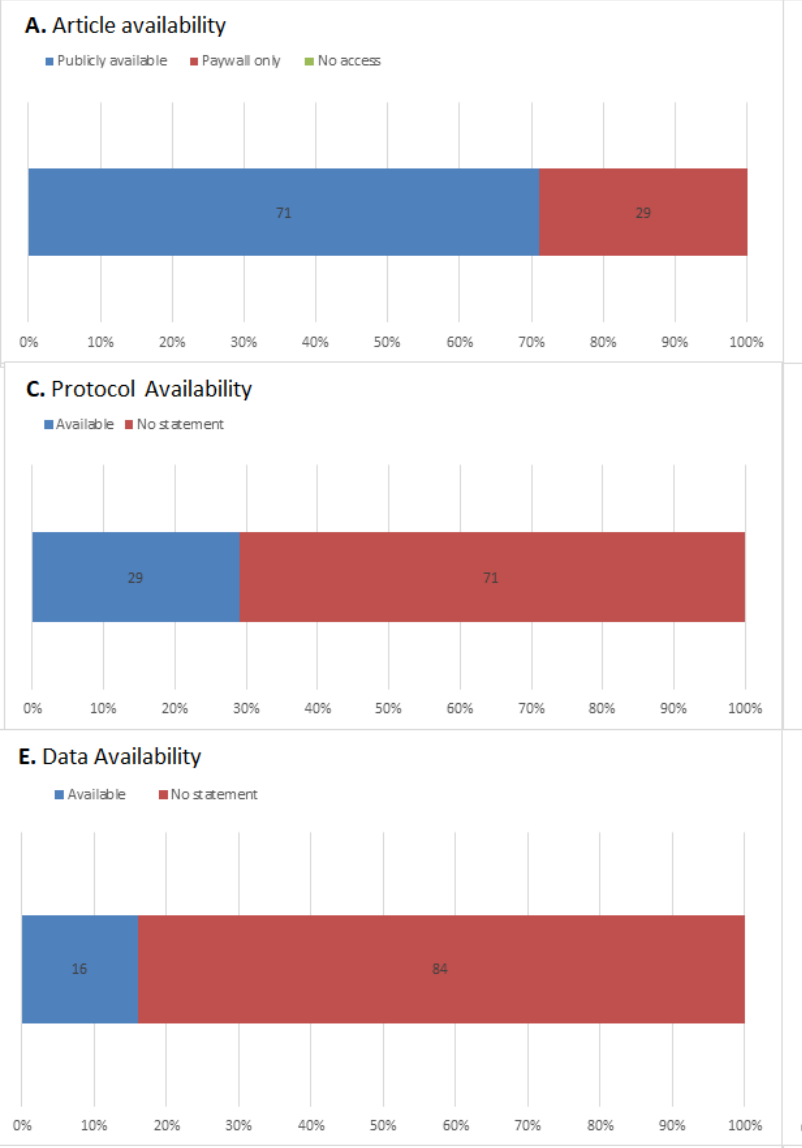

G. Replication Study

B. Pre-registration

m-Pre-registered $\boldsymbol{m}$ No sctcement $\boldsymbol{m}$ Not pre-registered
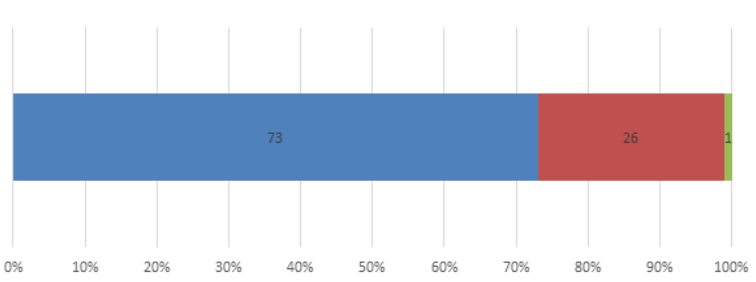

D. Material Availability

- Available no No stament

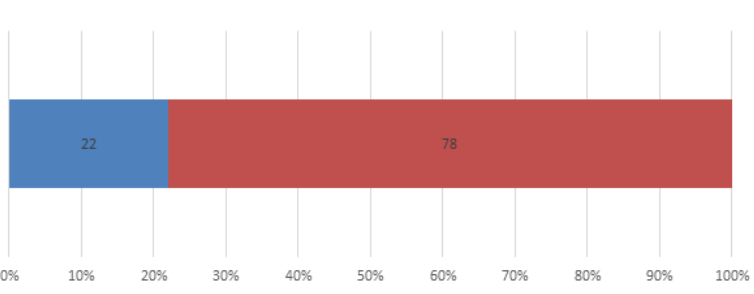

F. Analysis Script Availability

mavailable $\quad$ Nostatement

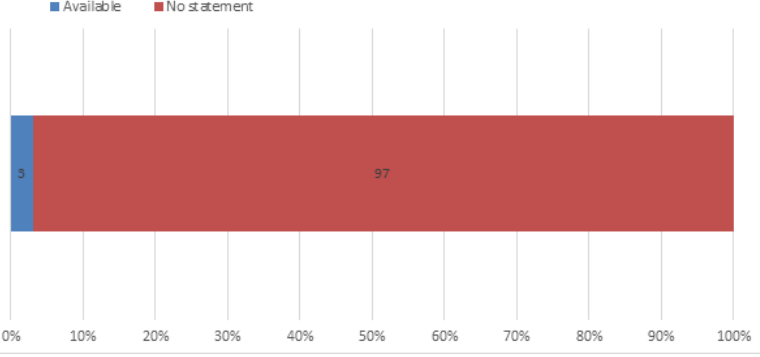

H. Funding Statement

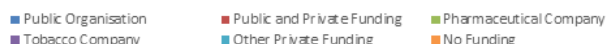
- No

=No Funding

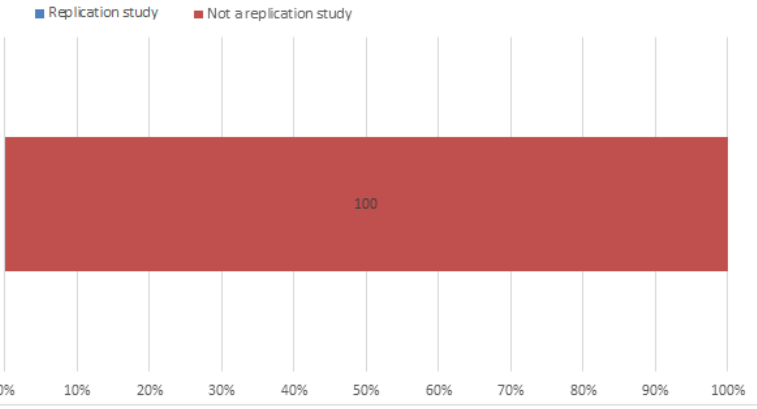

I. Conflicts of Interest

- Confict $\quad$ - Noconflict $\mid$ Nostament
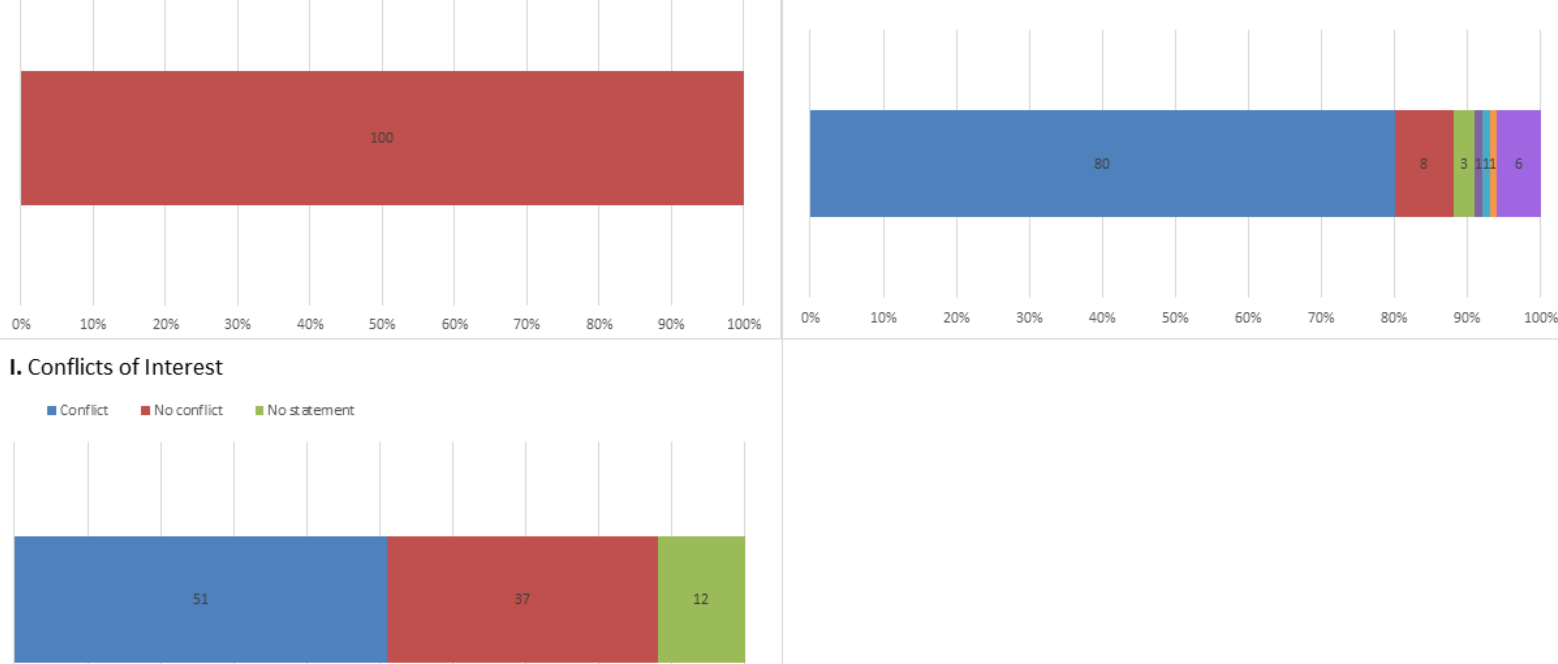